\section{GTB-3550 TRI-SPECIFIC KILLER ENGAGER TRIKETM DRIVES NK CELLS EXPANSION AND CYTOTOXICITY IN ACUTE MYELOID LEUKEMIA (AML) AND MYELODYSPLASTIC SYNDROMES (MDS) PATIENTS}

${ }^{1}$ Martin Felices, ${ }^{1}$ Erica Warlick, 'Mark Juckett, 'Daniel Weisdorf, 'Daniel Vallera, ${ }^{1}$ Sarah Miller, ${ }^{1}$ Rose Wangen, 'Dixie Lewis, ${ }^{1} J$ oAnne Knox, ${ }^{2}$ Martin Schroeder, ${ }^{1}$ Jeffrey Miller*. 'University of Minnesota, Minneapolis, MN, USA; ${ }^{2}$ GT Biopharma, Beverly Hills, CA, USA

Background Treatment for relapsed/refractory $[\mathrm{r} / \mathrm{r}]$ AML and high risk MDS is dismal and NK cell infusions (with IL-2 or IL-15) after lymphodepleting chemotherapy can result in 30$40 \%$ remission. However, NK cells lack antigen specificity and require cytokine for expansion. We have developed a novel tri-specific molecule, ${ }^{1}$ termed GTB-3550 TriKE, comprised of IL-15 surrounded by two single chain variable fragments (scFvs), one against CD16 on NK cells and one against CD33 on blasts.

Methods Supported by pre-clinical data, adults with CD33+ malignancies (r/r AML or MDS) are eligible (NCT03214666). Correlative objectives include the number, phenotype (Flow and CyTOF), and function of NK cells before and after therapy.

Results Twelve patients have completed therapy at doses of 5$150 \mathrm{mcg} / \mathrm{kg} /$ day without dose limiting toxicity. Out of the 11 with post treatment disease assessment, 3 patients had blasts cells decreases of 33, 61 and 63\% at cohorts 25, 50, and 100 $\mathrm{mcg} / \mathrm{Kg} /$ day, respectively. One patient $(150 \mathrm{mcg} / \mathrm{Kg} /$ day $)$ was found to have a multilineage leukemia with a significant population of CD19+/CD33- blasts not affected but demonstrated a $50 \%$ decrease of on-target CD19-/CD33+ blasts.Correlative studies show dose-dependent NK cell activity across cohorts, primarily by a robust expansion of NK cells without CD16 loss (figure 1A). Expansion is NK cell specific with preferential Ki-67 expression on NK cells at day 22 on study (figure 1B). Using IL-15 detection as a measure of GTB-3550 in serum, we find a short half-life as predicted with no evidence of drug accumulation (figure 1C). Despite rapid clearance, patient mononuclear cells without further activation demonstrate dose-dependent enhanced cytotoxicity against CD33+ HL-60 targets at days 8,15 , and 22 post initial treatment, 3 days after last drug (figure 1D). To better predict factors in responsiveness, responder and non-responder patient blood (d22) was incubated with IL-15 and HL-60 cells (no GTB3550 added) and NK cell degranulation (CD107a) was measured (figure 1E). Results indicate that responders have higher degranulating than non-responders. To explore further, we carried out 42-antigen CyTOF analysis. Differential expression analysis (DEA) on mature NK cells $(\mathrm{CD} 56+\mathrm{CD} 16+)$ at day 22 showed increased expression of the maturation marker CD57 and activation receptor NKG2D, while showing decreased expression of inhibitory KIR (figure $1 \mathrm{~F}$ ).

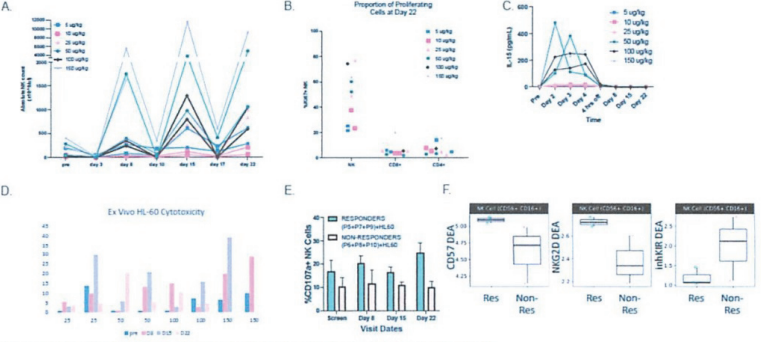

Abstract 444 Figure 1 Correlate studies outline potent, NK specific, activity of GTB-3550

Conclusions Our Phase I study demonstrates GTB-3550 TriKE safety, robust expansion of endogenous NK cells and a clinical signal of activity. Immune monitoring suggests that a schedule to maximize function in vivo with repeat courses will further enhance activity.

Trial Registration NCT03214666

\section{REFERENCES}

1. Vallera DA, Felices M, McElmurry R, McCullar V, Zhou X, Schmohl JU, et al. IL15 Trispecific Killer Engagers (TriKE) Make Natural Killer Cells Specific to CD33+ Targets While Also Inducing Persistence, In Vivo Expansion, and Enhanced Function. Clinical cancer research: an official journal of the American Association for Cancer Research 2016;22(14):3440-50.

Ethics Approval The protocol and consent procedures were approved by the University of Minnesota Institutional Review Board (HSC \# STUDY00000881). The study was approved by the FDA under BB-IND 136205. All patients and donors gave informed consent for treatment and prospective data collection in accordance with the Declaration of Helsinki.

http://dx.doi.org/10.1136/jitc-2021-SITC2021.444 